\section{0-67 DOES UNDERSTANDING OCCUPATIONAL MALE BREAST CANCER HELP US UNDERSTAND FEMALE BREAST CANCER?}

${ }^{1} J$ eavana Sritharan, Jill MacLeod, Mamadou Dakouo, Paul Demers, Chris McLeod. 'Ontario Health, Canada

\subsection{6/OEM-2021-EPI.12}

Objectives Identifying work-related risks for female breast cancer is often challenged by confounding hormonal and reproductive factors. Examining risk in men may help us understand occupational breast cancer risk factors, but few studies have been able to do this due to the rarity of male breast cancer. We used a large cohort of $2+$ million Ontario workers to determine if evidence of male breast cancer by occupation can inform our understanding of female breast cancer.

Methods Our study uses data from the Occupational Disease Surveillance System (ODSS), established through administrative data linkage, to follow 2,190,246 Ontario workers derived from WSIB lost time claims data (1983-2014). Breast cancer cases were identified in the Ontario Cancer Registry (OCR, 1964-2016). Cox-proportional hazard models were used to estimate age-adjusted hazard ratios (HR) and 95\% confidence intervals (CI) with an internal reference group of all other workers in the cohort.

Results A total of 17, 865 and 492 breast cancer cases were identified in working women and men, respectively. By occupation, elevated rates were observed for management/administration, social sciences, teaching and related, and medicine and health. Specifically, elevated rates were observed for elementary and secondary school teachers $(\mathrm{HRw}=1.27,95 \% \mathrm{CI}$ 1.19-1.35; $\mathrm{HRm}=2.16, \quad 95 \% \quad \mathrm{CI}=1.02-4.55)$, nurses $(\mathrm{HRw}=1.08,95 \% \mathrm{CI}=1.04-1.13 ; \mathrm{HRm}=4.73$, 95\% CI $=3.02-$ 7.41) and other medicine/health workers ( $\mathrm{HRw}=1.18,95 \%$ $\mathrm{CI}=1.03-1.34 ; \mathrm{HRm}=6.50,95 \% \mathrm{CI}=2.69-15.7)$.

Conclusion The ODSS enabled the analysis of a large cohort of working men and women with breast cancer. Findings show that at-risk groups were similar among both sexes, demonstrating the importance of occupation for breast cancer risk, although the HRs were much higher for men. Further research is needed in potential job-related factors such as sedentary behaviour, stress, shift work, and for some occupations, radiation exposure.

\section{0-233 OCCUPATIONAL SOLAR EXPOSURE AND RISK OF SUB- TYPES OF BREAST CANCER IN DANISH WOMEN}

${ }^{1} J o h n n i$ Hansen, Julie Pedersen, Katrine Strandberg-Larsen, Michael Andersson. 'Danish Cancer Society, Denmark

\subsection{6/OEM-2021-EPI.13}

Introduction It has been suggested that exposure to UV radiation from the sun (UVR) may increase the blood level of vitamin $\mathrm{D}$ and, in turn, decrease the risk of breast cancer.

Objectives We explored the associations between outdoor occupational solar radiation and subtypes of female breast cancer.

Methods Totally 38,375 female employees under the age 70 years were identified with first primary breast cancer from the Danish Cancer Registry. Five age matched employed controls, were, based on the incidence density principle, randomly chosen. The Danish Supplementary Pension Fund Register was used to retrieve full employment history. A job exposure matrix was used to assess individual occupational UVR exposure. Conditional logistic regression with adjustment for reproductive factors and SES was used to estimate odds ratios (ORs).

Results Longer duration of UVR exposure $(\geq 20$ years: $\mathrm{OR}=0.8,95 \% \mathrm{CI}: 0.75-0.92)$ and highest cumulative exposure $(\mathrm{OR}=0.9,95 \% \mathrm{CI}: 0.83-0.95)$ were inversely associated with the risk of breast cancer after age 50 years. Further, the reduced risk pattern for duration of exposure was most pronounced for estrogen receptor negative tumors $(\geq 20$ years: $\mathrm{OR}=0.8$, 95\% CI: 0.57-0.98).

Conclusion Our study of outdoor workers shows a modest decreased breast cancer risk.

\section{0-288 OCCUPATIONAL EXPOSURES AND BREAST CANCER RISK IN THE CECILE STUDY}

${ }^{1}$ Lisa Leung, Anita Koushik, Pascal Guenel. 'Université Paris-Saclay (CESP, Inserm) and Université de Montréal (CRCHUM), France

\subsection{6/OEM-2021-EPI.14}

Introduction The etiology of breast cancer is only partially understood. An increasing body of epidemiological evidence indicates that environmental and occupational factors may affect breast cancer risk, yet no established risk factors have been identified. Although recent studies have shown increased risks associated with specific workplace exposures, the evidence remains largely inconclusive for most occupational agents.

Objectives To examine associations between selected occupational exposures and breast cancer risk.

Methods In a population-based case-control study conducted in France between 2005 and 2008, detailed information on lifetime occupational history was collected for 1,206 cases and 1,294 population controls. An industrial hygienist coded occupations and industries for each job held by a participant. To identify occupational exposures, job codes were linked to the Canadian job-exposure matrix. Twenty-seven agents with relatively high prevalence were selected. Three exposure metrics of ever exposure, duration of exposure, and cumulative exposure to selected agents were analyzed. The reference group were participants having never been exposed to the specific agent. Odds ratios (OR) and 95\% confidence intervals for associations with breast cancer were estimated using logistic regression models, adjusting for well-established breast cancer risk factors.

Results Increased risks were suggested for high cumulative exposure to calcium carbonate, polyester fibres, fabric dust, cotton dust, aliphatic aldehydes, mononuclear aromatic hydrocarbons, and synthetic adhesives, with ORs ranging from 1.45 to 1.66. Inverse associations were observed for all exposure metrics for ultraviolet radiation and grain dust, with ORs ranging from 0.41 to 0.68 .

Conclusion These findings suggest that some occupational exposures may increase breast cancer risk. The decreased ORs associated with ultraviolet radiation and grain dust suggest that certain exposures that are typical of agricultural workers might be protective but should be interpreted with care. More research contributing to the knowledge base on occupational factors in relation to breast cancer is required. 